\title{
FRACTAL DIMENSION AS A SYMMETRY MEASURE IN 3D BRAIN MRI ANALYSIS
}

\author{
SURANI ANURADHA JAYASURIYA, ALAN WEE-CHUNG LIEW \\ School of Information and Communication Technology, Griffith University, Southport, QLD, Australia \\ E-MAIL: surani.jayasuriya @griffithuni.edu.au, a.liew@griffith.edu.au
}

\begin{abstract}
:
In brain image analysis, the automatic identification of symmetry plane has various applications. This paper presents a new method that uses the concept of fractal dimension as a quantitative measure for identifying symmetry plane in three-dimensional (3D) brain magnetic resonance (MR) images. The method was tested on various 3D MRI datasets. Robust and accurate results were obtained in our experiments.
\end{abstract}

Keywords:

Brain symmetry; Fractal Dimension; Magnetic Resonance Imaging; Mid-sagittal plane

\section{Introduction}

The human brain exhibits approximate bilateral symmetry. The plane that separates the left and right hemispheres is called the mid-sagittal plane (MSP) as it lines up with the sagittal plane of the human body. Brain symmetry plane is generally approximated by MSP (See Figures 1 and 2). Identification of the MSP is an important step in brain image analysis. Accurately located MSP can facilitate defining symmetrical regions of interest in the left and right hemispheres. It can help in identifying brain pathologies, like tumor and atrophy (volume loss) by comparing to healthy regions of the brain. Manual measurements are usually done in axial or coronal slices by a neurologist (See Figure 2). The large amount of scans makes this task tedious and time-consuming. An explicit automatic method for extracting MSP from the whole 3D image can become helpful.

Despite the variety of methods being proposed, MSP detection remains a challenging issue. Most of the existing work has concentrated on analyzing the image intensity, rather than the structure of the brain to detect symmetry. However, especially in MRI, artifacts like intensity non-uniformity (INU) give rise to smooth intensity variation across the image which can have a major impact on intensity based methods [1,2]. Additionally, the brain has a complex geometry which cannot be well described by Euclidean geometry. However, fractal geometry which can be expressed as an extension of Euclidean geometry offers the ability to characterize the complexity of objects.

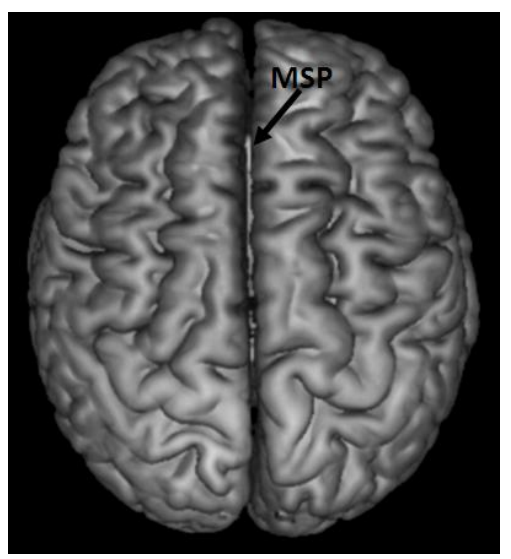

Figure1. Mid-sagittal plane

Fractal geometry, introduced by Mandelbrot [3], is widely used in many areas such as science, engineering, and medicine [4]. Fractal objects have a property called self-similarity. A self-similar object appears quantitatively the same, irrespective of its scale. Fractal analysis is already applied successfully to differentiate pathological tissues ([5-8]). Fractal dimension provides a way to quantify the shape complexity of objects using a single numerical value. It has proven to be a useful measure in describing the structure of a wide range of mathematical and naturally occurring objects $[5,9]$.

In this paper, we propose fractal dimension based symmetry index for detecting MSP directly from 3D MRI. While most of the existing methods in MSP detection are based on 2D projections of 3D MRI, our method considers the whole information in the $3 \mathrm{D}$ volume. Moreover, our method is robust to asymmetry present in the brain due to pathologies. 


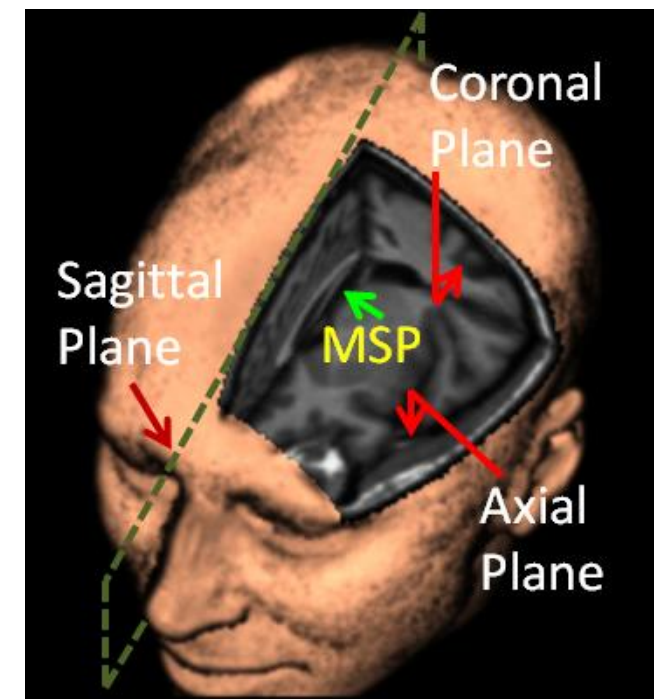

Figure2. MSP in different cross-sections of the brain

\section{Related Work}

A number of papers have dealt with the brain symmetry plane estimation problem. These methods can be divided into two main categories. Methods $([10,11])$ in the first category detect some anatomical feature (usually, the longitudinal fissure) to estimate the location of MSP. Methods ([12-15]) in the second category define MSP as a plane that maximizes a symmetry criterion which measures the similarity of two hemispheres.

Since brain is not perfectly symmetrical, various measures that quantify the degree of similarity between the two hemispheres have been proposed in the literature. Most of the work is based on cross correlation ([12,14,15]). Cross correlation $c c$ between two images $P$ and $Q$ can be calculated as

$$
c c=\frac{\sum_{i}\left(P_{i}-\bar{P}\right)\left(Q_{i}-\bar{Q}\right)}{\sqrt{\sum_{i}\left(P_{i}-\bar{P}\right)^{2}} \sqrt{\sum_{i}\left(Q_{i}-\bar{Q}\right)^{2}}}
$$

where $P_{i}$ and $Q_{i}$ are the intensity values in the $i$ th pixel and $\bar{P}$ and $\bar{Q}$ are the respective means of the entire image.

Usually, the image is reflected across the estimated plane and the $\mathrm{cc}$ is measured between the original and the reflected images. Besides being computationally demanding, cross correlation approach is highly sensitive to asymmetry in brain images.

Volkau et al.[11] proposed another symmetry measure using the Kullback-Leibler (KL) Divergence. Assuming that the MSP contains maximum amount of cerebrospinal fluid (CSF) amongst all sagittal slices, they have used KL divergence measure as a relative measure for comparing CSF. Probability distributions of intensities of two sagittal slices are taken as $\mathrm{P}=\left\{\mathrm{p}_{\mathrm{i}}\right\}$ and $\mathrm{Q}=\left\{\mathrm{q}_{\mathrm{i}}\right\}$. KL divergence measure is defined as

$$
\begin{aligned}
D_{K L}(p \| q) & =\sum_{i} p_{i} \log \left(p_{i}\right)-\sum_{i} p_{i} \log \left(q_{i}\right) \\
& =\sum_{i} p_{i} \log \left(p_{i} / q_{i}\right)
\end{aligned}
$$

The MSP is taken as the sagittal slice that gives the maximum KL divergence measure.

Another variation ([16]) based on KL divergence measure is Jeffrey's divergence that measures the similarity between the intensity distributions of the left and right parts of a brain. It is defined as

$$
\begin{aligned}
& J(p, q)=\sum_{i} p_{i} \log \left(p_{i} / q_{i}\right)+\sum_{i} q_{i} \log \left(q_{i} / p_{i}\right) \text { (3) } \\
& \mathrm{p}_{\mathrm{i}}=\text { (Number of voxels of intensity } i \text { in the left } \\
& \text { part)/(Total number of voxels in the left part) } \\
& \mathrm{q}_{\mathrm{i}}=\text { (Number of voxels of intensity } i \text { in the right } \\
& \text { part)/(Total number of voxels in the right part) }
\end{aligned}
$$

In their study, J-Divergence has been used as a quantitative measure of symmetry. The smaller the value, the more symmetrical the hemispheres are.

In this paper, we use fractal dimension as a symmetry measure for detecting MSP. The idea behind our approach is based on the assumption that the left and right hemispheres would be similar in structural complexity.

\section{Methodology}

\subsection{Fractal dimension}

Fractal geometry is considered as an extension of Euclidean geometry. A fractal is a complex geometrical object. The properties of Fractals are self-similarity and non-integer fractal dimension. A self-similar object appears qualitatively the same, irrespective of the magnification. For instance, the Sierpinski fractal is made by dividing a triangle into three equal triangles by shrinking the height and width of the triangle by two (See Figure 3).

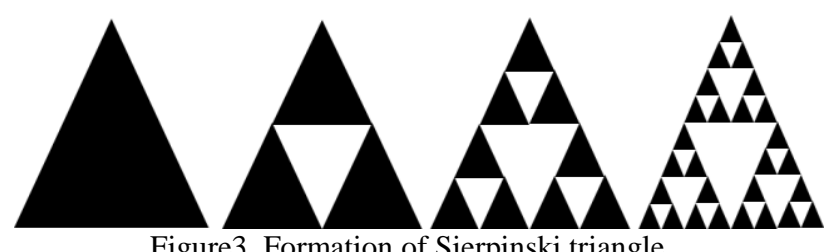

Figure3. Formation of Sierpinski triangle 
Fractal dimension (FD) can quantify the complexity of an object, indicating how much space is filled with increasing scale [3]. FD is a real number that describes the structural complexity of an object. It is defined as the exponent of the number of self similar pieces with magnification factor into which a figure may be broken. The equation can be written as follow.

$$
F D=\frac{\log (\text { number of self similar pieces })}{\log (\text { magnification factor })}
$$

For example, the Sierpinski triangle shown in Figure 3 is consisted of three self-similar triangles and each has a magnification factor of two. Therefore, its FD is

$$
F D=\frac{\log (3)}{\log (2)} \approx 1.58
$$

This suggests that fractal geometry might provide a useful quantitative measure for analyzing complex objects. The fact that medical images are complex in nature and exhibit some similarity in different scales has made fractal geometry useful in analyzing a variety of medical images [4]. FD has played an important role in various areas of medical and health research [7]. It has also been successfully used in quantifying morphological changes in neuroimages [8].

\subsection{Calculation of fractal dimension}

There are several methods for measuring fractal dimension. Many studies have adopted the box counting method [17] to measure the FD due to its simplicity and applicability to objects with or without self similarity. In the box counting method the image is covered with 3D boxes, each of side length $r . r$ is then progressively increased. At each scale, the number of boxes $N(r)$ needed to cover the whole structure is counted. Fractal dimension FD is defined as

$$
F D=-\frac{\ln N}{\ln r}
$$

The logarithm of $\mathrm{N}(\mathrm{r})$ versus the logarithm of $r$ gives a line whose gradient corresponds to FD (See Figure 4).

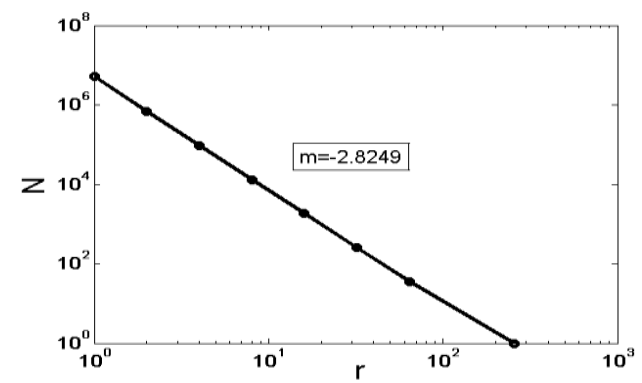

Figure 4. Log-log plot N vs. r

\subsection{Procedure}

Our approach is based on the assumption that the same FD holds for both left and right hemispheres. Once the 3D brain is divided into two parts in the sagittal direction, the difference of FD of the two parts should be at a minimum when the location of division is chosen at the MSP. Figure 5 demonstrates the log-log plots taken at any random sagittal location (left image) and at the MSP ground truth (right image). At the location of the ground truth, the left and right plots nearly coincide.

When the FD of the whole image, the left part, and the right part are denoted as $F D_{W}, F D_{L}$, and $F D_{R}$, respectively, we define a symmetry criterion $\alpha$ as

$$
\alpha=\frac{\left|F D_{L}-F D_{R}\right|}{F D_{W}}
$$
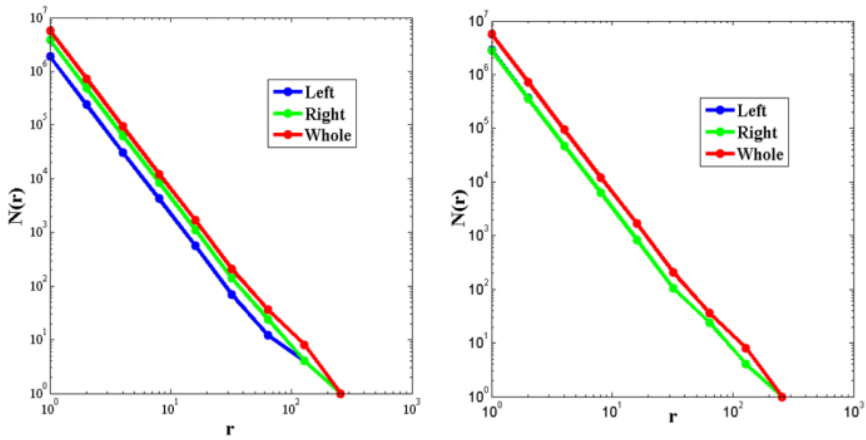

Figure 5. Log-log plots of N(r) vs. r. Left: Random sagittal location. Right:Ground truth MSP location

Our box counting algorithm and MSP detection algorithm are as follow.

BEGIN Box-Counting

- Initialize the cube sizes $r$

- FOR each cube size $r, r$ increasing at each iteration

- Count the number of cubes $N(r)$ needed to cover the 
image.

- $\quad$ END FOR

- Estimate FD as the slope of the least squares linear fit to the data

END

\section{BEGIN MSP Detection}

- Read 3D brain data $I(x, y, z)$. X, Y, Z be the left-right, anterior-posterior and top-bottom axes, respectively.

- Estimate the FD, $F D_{W}$ for whole image.

- Initialize the sagittal region of the image $I$ by ignoring the extreme $20 \%$ end slices.

- FOR each sagittal location $c$ in the region

- $\quad$ Estimate the $\mathrm{FD}, F D_{L}$ for $I(1: c)$.

- $\quad$ Estimate the FD, $F D_{R}$ for $I(c+1, n)$.

- Compute $\alpha(c)$ using $F D_{W}, F D_{L}$, and $F D_{R}$.

- ENDFOR

- Find the $c_{0}$ that gives the minimum $\alpha$

- Assign $c_{0}$ to be the sagittal location for MSP.

END

We tested the accuracy of our method by applying the algorithm on a mathematical fractal image 'Sierpinski Triangle'. Figure 6 shows the results.
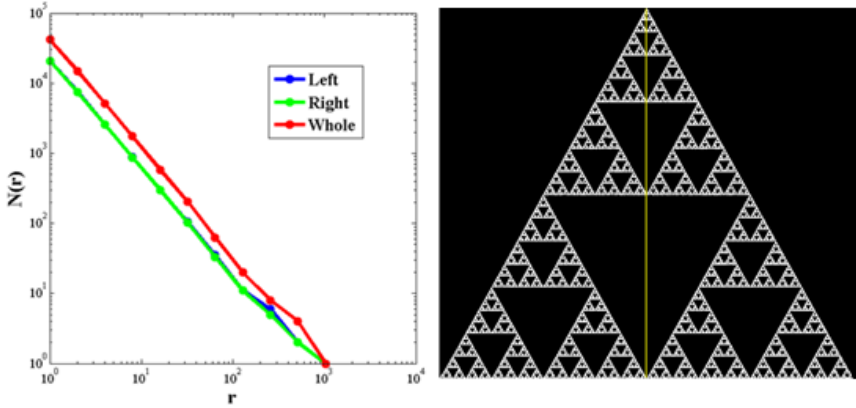

Figure 6. Left: Log-log plot of Sierpinski triangle. Right:Symmetry plane.

\section{Results}

The MR image datasets were obtained from IBSR (http://www.cma.mgh.harvard.edu/) and BrainWeb (http://www.bic.mni.mcgill.ca/brainweb/).

We considered $15 \mathrm{MR}$ scans of normal and pathological cases. T1-, T2- and PD-weighted images were included in the dataset. Phantom data from BrainWeb were used to analyze the effect of noise and intensity non-uniformity. The size of each 3D image was $181 \times 217 \times 181$. The robustness of the algorithm to the modality (T1, T2 and PD) of the image was also checked. Figures 7-10 shows some of the results obtained projected on the axial or coronal slices.

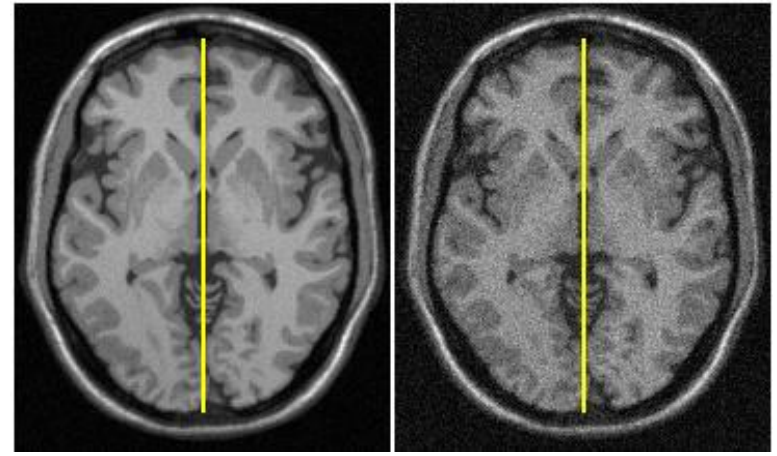

Figure 7. T1-weighted images

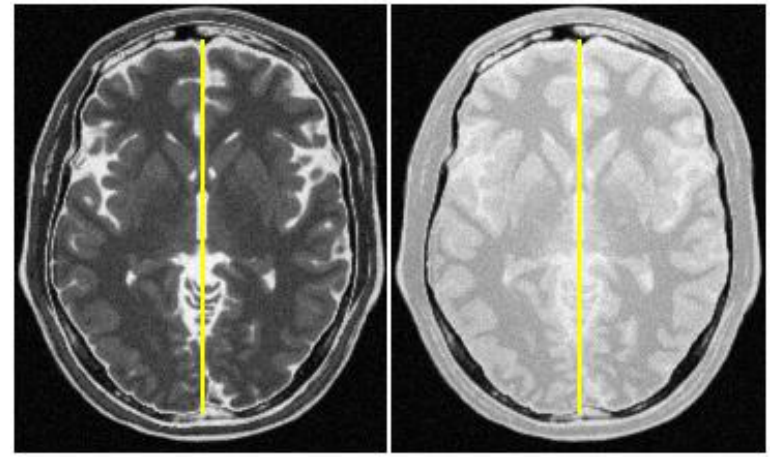

Figure 8. Left:T2-weighted image. Right: PD-weighted image
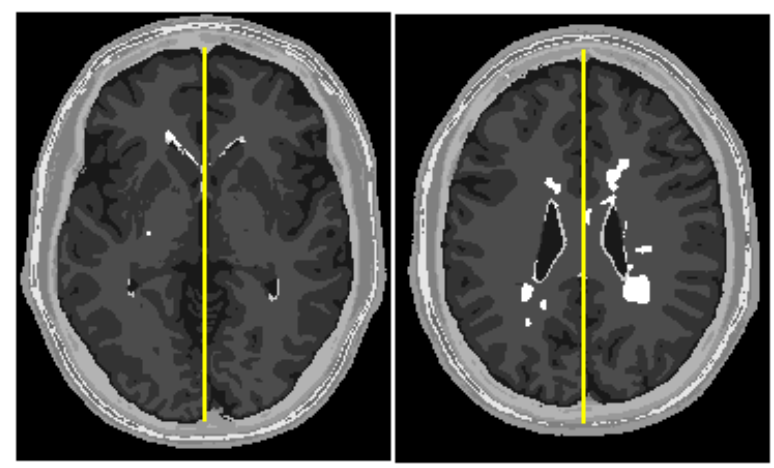

Figure 9. Left:Minor lesion. Right:Major lesion
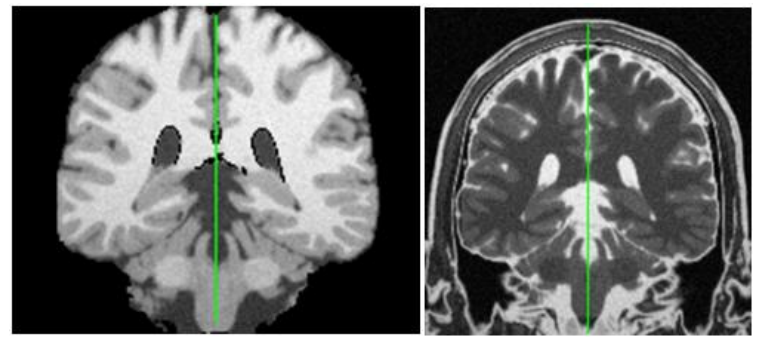

Figure 10. Coronal projection. Left:T1-weighted image Right:T2-weighted image 


\subsection{Visual evaluation}

Results were evaluated by comparing with manually detected MSP locations by an expert. For each image, we asked the expert to manually draw the MSP as a straight line. Comparison with our results indicated that all our results were highly accurate. The method was also found to be robust to noise, INU and different imaging modalities. We observed that our method gives good results in pathological cases as well. We postulate that this is because in the pathological brain the abnormality resides only in a small area and it does not affect the overall results when box-counting method is used.

\subsection{Comparison with other techniques}

Since cross-correlation is one of the most commonly used techniques in MSP detection algorithm, we compared the performance of our method with the cross-correlation method.

For each image we computed the cross-correlation (cc) of the original image and the reflected image about our estimated MSP plane. Then, for each image we rotated a line passing through the centroid in 1 degree intervals and computed the $c c$ as described in [12]. The line that gave the maximum $c c$ was compared with the line estimated by our method. Our approach consistently showed visually better results, despite its slightly lower $c c$ value. The results (Figures 11-14) show the superiority of our method compared with the cross-correlation based approach.

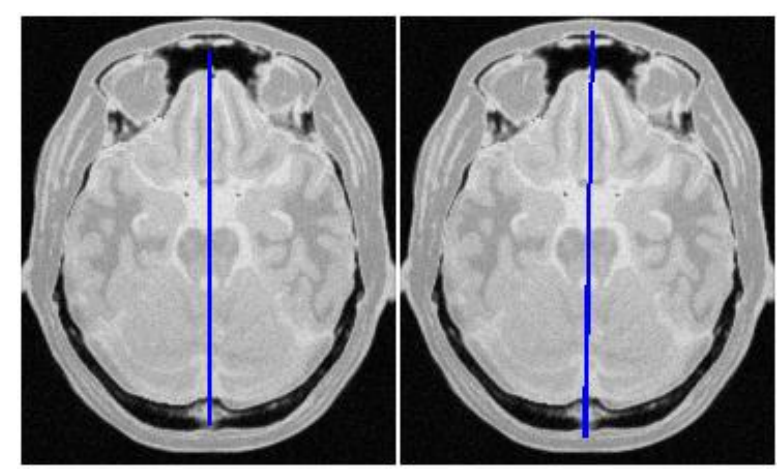

Figure 11. PD-weighted image Left: Our method (cc=0.9118)

Right: $\mathrm{cc}$ method $(\mathrm{cc}=0.9266)$

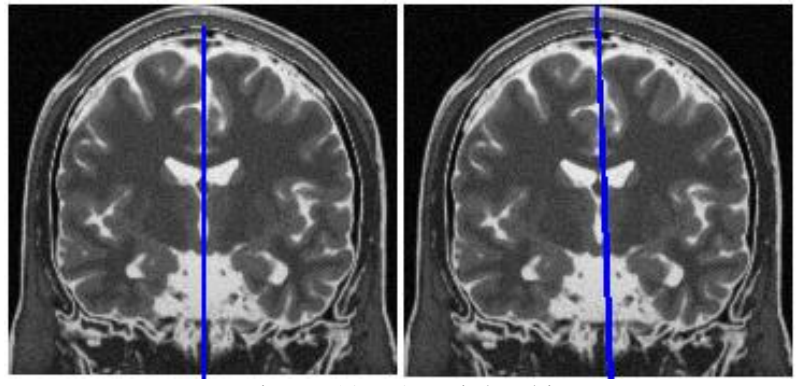

Figure 12. T2-weighted image Left: Our method $(\mathrm{cc}=0.6678)$

Right: $\mathrm{cc}$ method (cc=0.7028)

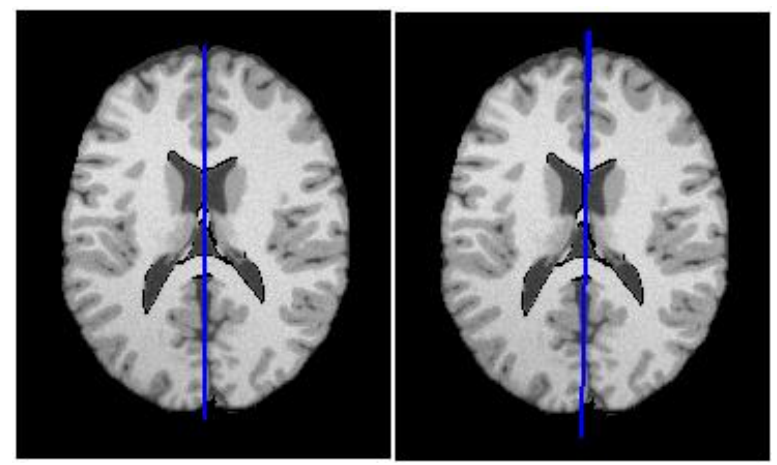

Figure 13. T1-weighted image Left: Our method $(\mathrm{cc}=0.9408)$

Right: $\mathrm{cc}$ method ( $\mathrm{cc}=0.9437)$
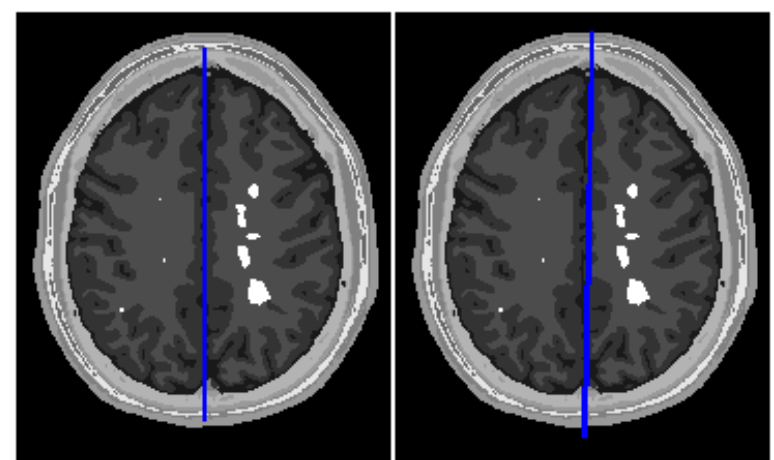

Figure 14. Image with lesion (multiple sclerosis) Left: Our method $(\mathrm{cc}=0.7849)$

Right: $\mathrm{cc}$ method $(\mathrm{cc}=0.8106)$ 


\section{Conclusions}

This paper proposed a novel method based on fractal dimension for automatically detecting the symmetry plane in 3D MR images. The method was tested on various 3D MR images in different imaging modalities. Excellent results were obtained. The method is also robust with respect to strong noise, intensity non-uniformity and pathology in the brain. In our future work, we plan to use the location of symmetry plane for asymmetry analysis of pathological brains. We also like to mention that FD is only one measure of fractal analysis. Although it can characterize the space-filling capacity, it cannot give further information on textural homogeneity of the surface. Other fractal measurements like lacunarity can be utilized to fully describe the complexity of the image.

\section{Acknowledgements}

This work was supported by the Australian Research Council (ARC) Discovery Grant DP1097059 and the Australian Postgraduate Award (APA).

\section{References}

[1] A. W. C. Liew and H. Yan, "An adaptive spatial fuzzy clustering algorithm for MR image segmentation", IEEE Transactions on Medical Imaging, 22(9):1063-1075, 2003. [2] A. W. C. Liew and H. Yan, "Current methods in the automatic tissue segmentation of 3D magnetic resonance brain images", Current Medical Imaging Reviews, 2(1):91-103, 2006.

[3] B. Mandelbrot, The Fractal Geometry of Nature, W.H.

Freeman and Company, 1982.

[4] R. Lopes and N. Betrouni, "Fractal and multifractal analysis: A review", Medical Image Analysis, 13:634-649, 2009.

[5] K. Iftekharuddin, "Techniques in fractal analysis and their applications in brain MRI", Medical imaging systems: technology and applications, Analysis and Computational Methods, 1:63-86, 2005.

[6] J. Blackledge and D. Dubovitskiy, "Object detection and classification with applications to skin cancer screening", ISAST Transactions on Intelligent Systems, 1(2):34-45, 2008. [7] T. Takahashi, H. Kosaka, T. Murata, M. Omori,K. Narita, H. Mitsuya, K. Takahashi, H. Kimura, and Y.Wada, "Application of a multifractal analysis to study brain white matter abnormalities of schizophrenia on t2-weighted magnetic resonance imaging", Psychiatry Research Neuroimaging, 171:177-188, 2009.
[8] S. Free, S. Sisodiya, M. Cook, D. Fish, and S. Shorvon, "Three-dimensional fractal analysis of the white matter surface from magnetic resonance images of the human brain", Cerebral Cortex, 6(6):830-836, 1996.

[9] K. Uemura, H. Toyama, S. Baba, Y. Kimura, M. Senda, and A. Uchiyama. Generation of fractal dimension images and its application to automatic edge detection in brain MRI. Computerized Medical Imaging and Graphics, 24:73-85, 2000.

[10] Q. Hu and W. Nowinski, "A rapid algorithm for robust and automatic extraction of the midsagittal plane of the human cerebrum from neuroimages based on local symmetry and outlier removal", NeuroImage, 20:2153-2165, 2003.

[11] I. Volkau, P. Bhanu, A. Ananthasubramaniam, A. Aziz, and W. Nowinski, "Extraction of the midsagittal plane from morphological neuroimages using the kullback-Leibler's measure", Medical Image Analysis, 10:863-874, 2006.

[12] Y. Liu, R. Collins, and W. Rothfus, "Robust midsagittal plane extraction from normal and pathological 3-D neuroradiology images", IEEE Transactions on Medical Imaging, 20(3):175-192, 2001.

[13] J. P. Thirion, S. Prima, G. Subsol, and N. Roberts, "Statistical analysis of normal and abnormal dissymmetry in volumetric medical images", In IEEE Workshop on Biomedical Image Analysis, pages 74-83, 2000.

[14] S. Prima, S. Ourselin, and N. Ayache, "Computation of the mid-sagittal plane in 3D brain images", IEEE Transactions on Medical Imaging, 21:122-138, 2001.

[15] A. Tuzikov, O. Colliot, and I. Bloch, "Evaluation of the symmetry plane in 3D MR brain images", Pattern Recognition Letters, 24:2219-2233, 2003.

[16] I. Volkau, P. Bhanu, A. Ananthasubramaniam, V. Gupta, A. Aziz, and W. Nowinski, "Quantitative analysis of brain asymmetry by using the divergence measure : Normal pathological brain discrimination", Academic Radiology, 13(6):752-758, 2006.

[17] D. Russell, J. Hanson, and E. Ott, "Dimension of strange Attractors", Physical Review Letters, 45(14):1175-1178, 1980.

[18]C. Rorden and M. Brett, "Stereotaxic display of brain lesions”, Behavioural Neurology, 12:191-200, 2000. 